\title{
The Study on Evaluation of Outsize Flood Based on Flow Betweenness Centrality
}

\author{
Jie Liu ${ }^{1}$, Xuanhua Xu ${ }^{1}$, Bin Pan ${ }^{2}$ \\ ${ }^{1}$ School of Business, Central South University, Changsha 410083, China \\ ${ }^{2}$ Institute of Financial Research, Wenzhou University, Wenzhou 325027, China

\section{基于改进流介数指标的重大洪港灾害社会稳定 风险评价研究 \\ 刘洁 ${ }^{1}$, 徐选华 ${ }^{1}$, 潘森 ${ }^{2}$ \\ 1 中南大学商学院, 长沙 410083 , 中国 \\ ${ }^{2}$ 温州大学金融研究院, 温州 100875, 中国}

\begin{abstract}
Aiming at the risk problems of social stability caused by outsize flood, on the base of the topology map for the evolution of flood social stability risk, we should focus on the position of the risk in the topology map for the evolution of flood social stability risk. The flow betweenness centrality is improved to evaluate the outsize social stability risk. From the evaluation results, we know that in the topology map for the evolution of flood social stability risk, human casualties, economic losses, lack of supplies, traffic congestion and interruption, public panic, gossip and group behavior are the most important.
\end{abstract}

Keywords: Flow betweenness centrality; Outsize floods; Society stability risk; Risk Evaluation

摘要: 针对重大洪涝灾害对社会稳定带来的风 险问题, 在灾害社会稳定风险拓扑结构的基础 上, 着眼于该风险在整个洪涝灾害社会稳定风 险结构中所处位置, 将复杂网络中的流介数指 标模型加以改进, 对重大洪涝灾害社会稳定风 险进行了评价。结果显示: 在整个洪涝灾害社 会稳定风险结构中, 人员伤亡、经济损失、物 资缺乏、交通受阻中断、社会大众恐慌、流言 及群体行为、洪涝灾害本身所处位置是最为重 要。

关键词: 流介数指标; 重大洪涝灾害; 社会稳 定风险; 风险评价
1. 引言

在重大洪涝灾害社会稳定风险研究的 过程中, 我们发现, 对重大洪涝灾害社会稳 定风险的有效预估, 恰恰是重大洪涝灾害社 会稳定风险应急防御的依据及关键程序。在 现实生活中, 因为种种原因对重大洪涝灾害 社会稳定风险的致灾能力及其灾害性后果 估计不足, 导致相关应急管理部门在应急服 务的过程中不能及时掌握重大洪涝灾害社 会稳定风险的发展态势, 对其可能导致的社 会影响情况及灾害严重程度失去掌控, 不能 形成充分、有效的应急联动, 从而影响了整 体防灾效果 ${ }^{[1]}$ 。北京“7 221 ” 暴雨就是一个比 较惨痛的教训, 尽管气象部门已经提前做了 “7.21”暴雨的预报, 但是对暴雨可能导致的 灾害后果估计不足, 使得灾前的转移处置、 隐患消除, 灾中的应急救援等工作做得不到 位, 而“7.21”暴雨出现了比较严重的人员伤 亡及财产损失, 北京市门头沟区政府在“7.21” 暴雨中提前做好了灾前应对措施, 取得了不 错的减灾成果, 全区无一人因灾死亡。随着 全球气候变化的不断加剧以及社会的快速 发展, 洪涝灾害发生更加频繁, 使得我国重 大洪涝灾害社会稳定风险呈现出复杂性、多 样性和放大性的特点, 其影响范围也逐渐扩 大。近年来, 中国不断发生重大洪涝灾害, 对社会稳定造成了严重的影响, 洪涝灾害社 会稳定风险已经成为制约社会稳定发展的 重要障碍因子。如何对重大洪涝灾害社会稳 
定风险进行评估分析, 已经成为重大洪涝灾 害社会稳定风险研究领域亟需解决的热点 问题 ${ }^{[2]}$ 。

对于重大洪涝灾害造成的社会稳定风 险进行全面的认识和评估, 对于防灾减灾工 作来说既是基础环节之一, 也是进行可持续 发展战略的迫切需求。然而, 对于洪涝灾害 社会风险的不确定性、危害性和复杂性等静 态特性是现阶段自然灾害风险研究的重点, 较少有人对洪涝灾害社会风险包含的潜在 动态性特征进行研究, 即风险并不是静止不 动的, 它会随时间与空间的变化而发生变化。 重大洪港灾害社会风险演变是一个由多种 影响因素构成的复杂系统过程, 涉及自然、 经济、人文等许多领域, 各因素对于风险演 变的作用方式、范围和强度也不同, 由于在 时空尺度上具有交叉叠合的特点, 从而导致 重大洪涝灾害社会风险演变过程变得更加 复杂和多样化。各种自然因素和人为因素一 直影响着重大洪涝灾害社会稳定风险, 因此 需要随着环境和条件的变化而不定期的对 洪涝灾害社会风险进行评价。例如, 气候变 化及损失大小的改变、社会经济的高速增长、 对洪涝灾害社会风险区划图精度要求的提 高等, 都需要重新对洪涝灾害社会风险进行 评价和区划。由于洪涝灾害社会风险评价是 一个不断更新、不断变化的动态过程, 当孕 灾环境发生重大变化或者承灾体的承载能 力具有明显的提高时, 就需要重新评估风险。 科学并及时的进行灾害社会风险评价, 正确

\section{2. 重大洪涝灾害社会稳定风险拓扑结构建} 模

重大洪涝灾害社会稳定风险系统结构 是由洪涝灾害直接引发的经济损失、房屋倒 塌、电力设施破坏、交通受阻中断、通讯中 断、农作物受损及其引发的一系列次生社会 稳定风险构成。本文在对湖南省怀化市 2013 年 8 月的特大洪涝灾害调研基础上, 将调研 资料整理分析之后, 再综合文献的研究, 确 立 22 个社会稳定风险及各个风险之间的出 入关系, 给出了重大洪涝灾害社会稳定风险 拓扑结构图, 如图 1 所示。

重大洪涝灾害社会稳定风险拓扑结构
由于在时空尺度上具有交叉叠合的特点, 从 而导致重大洪涝灾害社会风险演变过程变 得更加复杂和多样化。各种自然因素和人为 因素一直影响着重大洪涝灾害社会稳定风 险, 因此需要随着环境和条件的变化而不定 期的对洪涝灾害社会风险进行评价。例如, 气候变化及损失大小的改变、社会经济的高 速增长、对洪涝灾害社会风险区划图精度要 求的提高等, 都需要重新对洪涝灾害社会风 险进行评价和区划。由于洪涝灾害社会风险 评价是一个不断更新、不断变化的动态过程, 当孕灾环境发生重大变化或者承灾体的承 载能力具有明显的提高时, 就需要重新评估 风险。科学并及时的进行灾害社会风险评价, 正确认识风险随时间而发生变化的特征具 有重要的现实意义, 并将有助于科学有效的 制定灾害社会风险管理措施。

中共有 22 个节点和 39 条有向边。其中 22 个节点代表 22 个社会稳定风险事件, 其中 包括始端节点 (没有入度) 洪涝灾害、末端 节点 (没有出度) 流言及群体行为以及处于 始端节点和末端节点之间的演化风险节点; 39 条有向边表明各个节点之间的相互演化 关系。

从图 1 中可以看出, 重大洪涝灾害将直 接造成经济损失、房屋倒塌、电力设施破坏、 交通受阻中断、通讯中断、农作物受损一系 列社会稳定风险; 进而造成交通事故、居民 居无定所、社会大众恐慌、市场供应紧张等 社会稳定风险; 最终引发物价上涨、流言及 群体行为、生产生活困难、哄抢购物等严重 的社会稳定风险, 各个风险之间的相互关系 使得风险之间相互影响。

\section{3. 流介数指标模型改进方法}

针对洪涝灾害风险分析和评价, 已开展 了大量深入的研究, 认为致灾因子、孕灾环 境和承灾体三者相互作用构成了洪水灾害 系统 ${ }^{[3-7]}$, 综合致灾因子和孕灾环境的自然 属性及承灾体的社会属性两方面来评价区 域洪水灾害的风险。另外, 洪灾风险评价方 法有指标体系评估法、模糊综合评判法、人 工神经网络、统计分析法等综合方法, 从洪 水灾害危险性和洪水灾害易损性两方面, 选 
Risk Analysis and Crisis Response in Big Data Era (RAC-16)

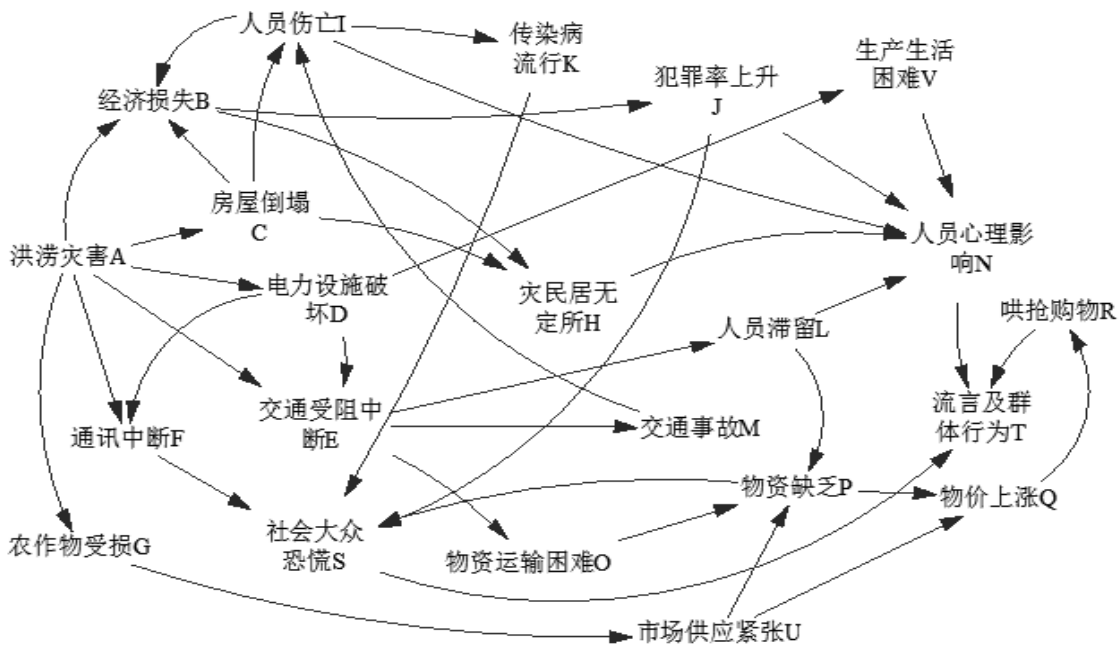

图 1 重大洪涝灾害社会稳定风险拓扑结构图

取评价指标和建立评价模型的评估方法已 较为成熟 ${ }^{[8-12]}$ 。黄民生等 ${ }^{[13]}$ 认为洪灾风险的 等级划分具有相对性和模糊性, 没有绝对统 一的定量指标, 所以对洪灾风险分级采用模 糊数学综合评判方法; 赖成光等 ${ }^{[14-15]}$ 利用 BP 网络处理非线性问题的优势, 提出基于 BP 神经网络建立洪灾风险综合评价模型; 针对评价指标的不确定性, 李帅杰等 ${ }^{[16-17]}$ 采用层次分析法 (AHP), 王兆礼等 ${ }^{[18-21]}$ 利用 熵权法, 以评价指标值构成的判断矩阵来确 定指标权重, 从而消除各指标权重的人为干 扰。灾害社会稳定风险评价方法研究相对较 少, 本文改进了流介数指标模型, 在此基础 上提出了一种重大洪涝灾害社会稳定风险 评价方法。

3.1.流介数指标模型

流介数指标（ Flow Betweenness Centrality) 是以去除最短路径的概念来定义 介数, 因而流介数指标能够确定整体上的几 何中心节点 ${ }^{[22-23]}$ 。某一节点 $x$ 的流介数指标 定义为:

$$
C_{B}(x)=\sum_{j<k} \frac{g_{j k}(x)}{g_{j k}}
$$

其中, $g_{j k}$ 表示节点 $j$ 和节点 $k$ 之间的
路径数, $g_{j k}(x)$ 表示节点 $j$ 和节点 $k$ 之间经

过节点 $x$ 的路径数。

3. 2. 流介数指标模型的改进

之前关于复杂网络流介数指标模型的 应用局限在结点之间无方向 (即无出入度之 别）的网络模型, 考虑到本文中复杂网络拓 扑结构中节点有方向, 将流介数指标模型进 行如下改进。

考虑网络节点 $x$ 有出入度之分, 故定义:

$$
\begin{aligned}
& C_{B}(x)_{1}=\frac{g_{j \rightarrow k}(x)}{g_{j \rightarrow k}} \\
& C_{B}(x)_{2}=\frac{g_{k \rightarrow j}(x)}{g_{k \rightarrow j}}
\end{aligned}
$$

$g_{j \rightarrow k}$ 和 $g_{k \rightarrow j}$ 分别表示节点 $j$ 到节点 $k$ 之间的路径数，节点 $k$ 到节点 $j$ 之间的路 径数; $g_{j \rightarrow k}(x)$ 和 $g_{k \rightarrow j}(x)$ 分别表示节点 $j$ 到节点 $k$ 之间经过节点 $x$ 的路径数, 节点 $k$ 到节点 $j$ 之间经过节点 $x$ 的路径数。

对于节点 $x$, 其在出度和入度所处的位 置重要程度相同, 所以我们改进节点 $x$ 的流 介数指标模型为: 
Risk Analysis and Crisis Response in Big Data Era (RAC-16)

$$
\begin{aligned}
C_{B}(x) & =\frac{1}{2}\left[C_{B}(x)_{1}+C_{B}(x)_{2}\right] \\
& =\frac{1}{2}\left[\frac{g_{j \rightarrow k}(x)}{g_{j \rightarrow k}}+\frac{g_{k \rightarrow j}(x)}{g_{k \rightarrow j}}\right]
\end{aligned}
$$

改进的流介数指标能够反映各个风险 节点之间的出入关系, 反映了风险节点 $x$ 在 整个重大洪涝灾害社会稳定风险结构中的 几何中心, 即风险节点 $x$ 在整个重大洪涝灾 害社会稳定风险中的风险损失程度。 $C_{B}(x)$
的值越大, 表明风险节点 $x$ 在整个重大洪涝 灾害社会稳定风险中造成的损害程度越大, 对风险发展态势的影响越大。

\section{4. 重大洪涝灾害社会稳定风险评价与分析}

将改进的流介数指标模型应用到重大洪 涝灾害社会稳定风险评价分析中, 经过对重 大洪涝灾害社会稳定风险拓扑结构图的分 析, 结合路径数 $g_{j \rightarrow k}$ 的定义, 可以得出相应 节点之间的路径数, 如下表 1 所示:

表 1 相应节点之间的路径数 $g_{j \rightarrow k}$

\begin{tabular}{|c|c|c|c|c|c|c|c|c|c|c|c|c|c|c|c|c|c|c|c|c|c|c|}
\hline A & - & 5 & 1 & 1 & 2 & 2 & 1 & 6 & 3 & 5 & 3 & 2 & 2 & 17 & 2 & 5 & 6 & 6 & 15 & 38 & 1 & 1 \\
\hline B & 0 & - & 0 & 0 & 0 & 0 & 0 & 1 & 0 & 1 & 0 & 0 & 0 & 2 & 0 & 0 & 0 & 0 & 1 & 3 & 0 & 0 \\
\hline $\mathrm{C}$ & 0 & 2 & - & 0 & 0 & 0 & 0 & 3 & 1 & 2 & 1 & 0 & 0 & 6 & 0 & 0 & 0 & 0 & 3 & 9 & 0 & 0 \\
\hline D & 0 & 1 & 0 & - & 1 & 1 & 0 & 1 & 1 & 1 & 1 & 1 & 1 & 5 & 1 & 2 & 2 & 2 & 5 & 12 & 0 & 1 \\
\hline E & 0 & 1 & 0 & 0 & - & 0 & 0 & 1 & 1 & 1 & 1 & 1 & 1 & 4 & 1 & 2 & 2 & 2 & 4 & 10 & 0 & 0 \\
\hline $\mathrm{F}$ & 0 & 0 & 0 & 0 & 0 & - & 0 & 0 & 0 & 0 & 0 & 0 & 0 & 0 & 0 & 0 & 0 & 0 & 1 & 1 & 0 & 0 \\
\hline $\mathrm{G}$ & 0 & 0 & 0 & 0 & 0 & 0 & - & 0 & 0 & 0 & 0 & 0 & 0 & 0 & 0 & 1 & 2 & 2 & 1 & 3 & 1 & 0 \\
\hline $\mathrm{H}$ & 0 & 0 & 0 & 0 & 0 & 0 & 0 & - & 0 & 0 & 0 & 0 & 0 & 1 & 0 & 0 & 0 & 0 & 0 & 1 & 0 & 0 \\
\hline I & 0 & 1 & 0 & 0 & 0 & 0 & 0 & 1 & - & 1 & 1 & 0 & 0 & 3 & 0 & 0 & 0 & 0 & 2 & 5 & 0 & 0 \\
\hline $\mathrm{J}$ & 0 & 0 & 0 & 0 & 0 & 0 & 0 & 0 & 0 & - & 0 & 0 & 0 & 1 & 0 & 0 & 0 & 0 & 1 & 2 & 0 & 0 \\
\hline $\mathrm{K}$ & 0 & 0 & 0 & 0 & 0 & 0 & 0 & 0 & 0 & 0 & - & 0 & 0 & 0 & 0 & 0 & 0 & 0 & 1 & 1 & 0 & 0 \\
\hline $\mathrm{L}$ & 0 & 0 & 0 & 0 & 0 & 0 & 0 & 0 & 0 & 0 & 0 & - & 0 & 1 & 0 & 1 & 1 & 1 & 1 & 3 & 0 & 0 \\
\hline $\mathrm{M}$ & 0 & 1 & 0 & 0 & 0 & 0 & 0 & 1 & 1 & 1 & 1 & 0 & - & 3 & 0 & 0 & 0 & 0 & 2 & 5 & 0 & 0 \\
\hline $\mathrm{N}$ & 0 & 0 & 0 & 0 & 0 & 0 & 0 & 0 & 0 & 0 & 0 & 0 & 0 & - & 0 & 0 & 0 & 0 & 0 & 1 & 0 & 0 \\
\hline $\mathrm{O}$ & 0 & 0 & 0 & 0 & 0 & 0 & 0 & 0 & 0 & 0 & 0 & 0 & 0 & 0 & - & 1 & 1 & 1 & 1 & 2 & 0 & 0 \\
\hline $\mathrm{P}$ & 0 & 0 & 0 & 0 & 0 & 0 & 0 & 0 & 0 & 0 & 0 & 0 & 0 & 0 & 0 & - & 1 & 1 & 1 & 2 & 0 & 0 \\
\hline $\mathrm{Q}$ & 0 & 0 & 0 & 0 & 0 & 0 & 0 & 0 & 0 & 0 & 0 & 0 & 0 & 0 & 0 & 0 & - & 1 & 0 & 1 & 0 & 0 \\
\hline $\mathrm{R}$ & 0 & 0 & 0 & 0 & 0 & 0 & 0 & 0 & 0 & 0 & 0 & 0 & 0 & 0 & 0 & 0 & 0 & - & 0 & 1 & 0 & 0 \\
\hline S & 0 & 0 & 0 & 0 & 0 & 0 & 0 & 0 & 0 & 0 & 0 & 0 & 0 & 0 & 0 & 0 & 0 & 0 & - & 1 & 0 & 0 \\
\hline $\mathrm{T}$ & 0 & 0 & 0 & 0 & 0 & 0 & 0 & 0 & 0 & 0 & 0 & 0 & 0 & 0 & 0 & 0 & 0 & 0 & 0 & - & 0 & 0 \\
\hline $\mathrm{U}$ & 0 & 0 & 0 & 0 & 0 & 0 & 0 & 0 & 0 & 0 & 0 & 0 & 0 & 0 & 0 & 1 & 2 & 2 & 1 & 3 & - & 0 \\
\hline $\mathrm{V}$ & 0 & 0 & 0 & 0 & 0 & 0 & 0 & 0 & 0 & 0 & 0 & 0 & 0 & 1 & 0 & 0 & 0 & 0 & 0 & 1 & 0 & - \\
\hline
\end{tabular}

\begin{tabular}{lllllllllllllllllllllll}
\multicolumn{11}{c|}{1} & 相应节点之间的路径数 $g_{j \rightarrow k}$ \\
\hline A & B & C & D & E & F & G & H & I & J & K & L & M & N & O & P & Q & R & S & T & U & V
\end{tabular}

表 2 节点 $j$ 和节点 $k$ 之间经过节点 $A$ 的路径数 $C_{B}(A)$

\begin{tabular}{llllllllllllllllllllllll}
\hline & $\mathrm{B}$ & $\mathrm{C}$ & $\mathrm{D}$ & $\mathrm{E}$ & $\mathrm{F}$ & $\mathrm{G}$ & $\mathrm{H}$ & $\mathrm{I}$ & $\mathrm{J}$ & $\mathrm{K}$ & $\mathrm{L}$ & $\mathrm{M}$ & $\mathrm{N}$ & $\mathrm{O}$ & $\mathrm{P}$ & $\mathrm{Q}$ & $\mathrm{R}$ & $\mathrm{S}$ & $\mathrm{T}$ & $\mathrm{U}$ & $\mathrm{V}$ \\
\hline $\mathrm{A}$ & 5 & 1 & 1 & 2 & 2 & 1 & 6 & 3 & 5 & 3 & 2 & 2 & 17 & 2 & 5 & 6 & 6 & 15 & 38 & 1 & 1
\end{tabular}

(2) 节点 $j$ 到节点 $k$ 之间经过节点 $B$ 的路 径数的相应数值, 如表 3 所示: 
Risk Analysis and Crisis Response in Big Data Era (RAC-16)

表 3 节点 $j$ 和节点 $k$ 之间经过节点 $B$ 的路径数 $C_{B}(B)$

\begin{tabular}{ccccccc}
\hline & $\mathrm{B}$ & $\mathrm{H}$ & $\mathrm{J}$ & $\mathrm{N}$ & $\mathrm{S}$ & $\mathrm{T}$ \\
\hline $\mathrm{A}$ & 4 & 5 & 5 & 11 & 5 & 16 \\
$\mathrm{~B}$ & - & 1 & 1 & 2 & 1 & 3 \\
$\mathrm{C}$ & 2 & 2 & 2 & 4 & 2 & 6 \\
$\mathrm{D}$ & 1 & 1 & 1 & 2 & 1 & 3 \\
$\mathrm{E}$ & 1 & 1 & 1 & 2 & 1 & 3 \\
$\mathrm{I}$ & 1 & 1 & 1 & 2 & 1 & 3 \\
$\mathrm{M}$ & 1 & 1 & 1 & 2 & 1 & 3
\end{tabular}

（3）节点 $j$ 到节点 $k$ 之间经过节点 $C$ 的路 径数的相应数值, 如表 4 所示:

表 4 节点 $j$ 和节点 $k$ 之间经过节点 $C$ 的路径数 $C_{B}(C)$

\begin{tabular}{cccccccccc}
\hline & $\mathrm{B}$ & $\mathrm{C}$ & $\mathrm{H}$ & $\mathrm{I}$ & $\mathrm{J}$ & $\mathrm{K}$ & $\mathrm{N}$ & $\mathrm{S}$ & $\mathrm{T}$ \\
\hline $\mathrm{A}$ & 2 & 1 & 3 & 1 & 2 & 1 & 6 & 3 & 9 \\
\hline
\end{tabular}

(4) 节点 $j$ 到节点 $k$ 之间经过节点 $D$ 的路 径数的相应数值，如表 5 所示

表 5 节点 $j$ 和节点 $k$ 之间经过节点 $D$ 的路径数 $C_{B}(D)$

\begin{tabular}{cccccccccccccc}
\hline & $\mathrm{D}$ & $\mathrm{E}$ & $\mathrm{F}$ & $\mathrm{L}$ & $\mathrm{M}$ & $\mathrm{N}$ & $\mathrm{O}$ & $\mathrm{P}$ & $\mathrm{Q}$ & $\mathrm{R}$ & $\mathrm{S}$ & $\mathrm{T}$ & $\mathrm{V}$ \\
\hline $\mathrm{A}$ & 1 & 1 & 1 & 1 & 1 & 2 & 1 & 2 & 2 & 2 & 3 & 7 & 1
\end{tabular}

(5) 节点 $j$ 到节点 $k$ 之间经过节点 $E$ 的路 径数的相应数值, 如表 6 所示:

表 6 节点 $j$ 和节点 $k$ 之间经过节点 $E$ 的路径数 $C_{B}(E)$

\begin{tabular}{cccccccccccccccc}
\hline & $\mathrm{B}$ & $\mathrm{E}$ & $\mathrm{H}$ & $\mathrm{I}$ & $\mathrm{J}$ & $\mathrm{K}$ & $\mathrm{L}$ & $\mathrm{M}$ & $\mathrm{N}$ & $\mathrm{O}$ & $\mathrm{P}$ & $\mathrm{Q}$ & $\mathrm{R}$ & $\mathrm{S}$ & $\mathrm{T}$ \\
\hline $\mathrm{A}$ & 2 & 2 & 2 & 2 & 2 & 2 & 2 & 2 & 8 & 2 & 4 & 4 & 4 & 8 & 20 \\
$\mathrm{D}$ & 1 & 1 & 1 & 1 & 1 & 1 & 1 & 1 & 4 & 1 & 2 & 2 & 2 & 4 & 10 \\
\hline
\end{tabular}

(6) 节点 $j$ 到节点 $k$ 之间经过节点 $F$ 的路 径数的相应数值，如表 7 所示:

\begin{tabular}{|c|c|c|c|}
\hline & $\mathrm{F}$ & $\mathrm{S}$ & $\mathrm{T}$ \\
\hline A & 2 & 2 & 2 \\
\hline D & 1 & 1 & 1 \\
\hline
\end{tabular}

(7) 节点 $j$ 到节点 $k$ 之间经过节点 $G$ 的路 径数的相应数值, 如表 8 所示:

表 8 节点 $j$ 和节点 $k$ 之间经过节点 $G$ 的路径数 $C_{B}(G)$

\begin{tabular}{cccccccc}
\hline & $\mathrm{G}$ & $\mathrm{P}$ & $\mathrm{Q}$ & $\mathrm{R}$ & $\mathrm{S}$ & $\mathrm{T}$ & $\mathrm{U}$ \\
\hline $\mathrm{A}$ & 1 & 1 & 2 & 2 & 1 & 3 & 1 \\
\hline
\end{tabular}

（8）节点 $j$ 到节点 $k$ 之间经过节点 $H$ 的路 $\quad$ 径数的相应数值，如表 9 所示: 
Risk Analysis and Crisis Response in Big Data Era (RAC-16)

表 9 节点 $j$ 和节点 $k$ 之间经过节点 $H$ 的路径数 $C_{B}(H)$

\begin{tabular}{llll}
\hline & $\mathrm{H}$ & $\mathrm{N}$ & $\mathrm{T}$ \\
\hline $\mathrm{A}$ & 6 & 6 & 6 \\
$\mathrm{~B}$ & 1 & 1 & 1 \\
$\mathrm{C}$ & 3 & 3 & 3 \\
$\mathrm{D}$ & 1 & 1 & 1 \\
$\mathrm{E}$ & 1 & 1 & 1 \\
$\mathrm{I}$ & 1 & 1 & 1 \\
$\mathrm{~N}$ & 1 & 1 & 1 \\
\hline
\end{tabular}

（9）节点 $j$ 到节点 $k$ 之间经过节点 $\mathrm{I}$ 的路径数的相应数值，如表 10 所示:

表 10 节点 $j$ 和节点 $k$ 之间经过节点 $\mathrm{I}$ 的路径数 $C_{B}(I)$

\begin{tabular}{ccccccccc}
\hline & $\mathrm{B}$ & $\mathrm{H}$ & $\mathrm{I}$ & $\mathrm{J}$ & $\mathrm{K}$ & $\mathrm{N}$ & $\mathrm{S}$ & $\mathrm{T}$ \\
\hline $\mathrm{A}$ & 3 & 3 & 3 & 3 & 3 & 9 & 6 & 15 \\
$\mathrm{C}$ & 1 & 1 & 1 & 1 & 1 & 3 & 2 & 5 \\
$\mathrm{D}$ & 1 & 1 & 1 & 1 & 1 & 3 & 2 & 5 \\
$\mathrm{E}$ & 1 & 1 & 1 & 1 & 1 & 3 & 2 & 5 \\
$\mathrm{M}$ & 1 & 1 & 1 & 1 & 1 & 3 & 2 & 5 \\
\hline$(10)$ & 节点 $j$ 到节点 $k$ 之间经过节点 $\mathrm{J}$ 的路 & 径数的相应数值, 如表 11 所示: &
\end{tabular}

表 11 节点 $j$ 和节点 $k$ 之间经过节点 $\mathrm{J}$ 的路径数 $C_{B}(J)$

\begin{tabular}{lllll}
\hline & $\mathrm{J}$ & $\mathrm{N}$ & $\mathrm{S}$ & $\mathrm{T}$ \\
\hline $\mathrm{A}$ & 5 & 5 & 5 & 10 \\
$\mathrm{~B}$ & 1 & 1 & 1 & 2 \\
$\mathrm{C}$ & 2 & 2 & 2 & 4 \\
$\mathrm{D}$ & 1 & 1 & 1 & 2 \\
$\mathrm{E}$ & 1 & 1 & 1 & 2 \\
$\mathrm{I}$ & 1 & 1 & 1 & 2 \\
$\mathrm{M}$ & 1 & 1 & 1 & 2 \\
\hline
\end{tabular}

(11) 节点 $j$ 到节点 $k$ 之间经过节点 $K$ 的路 径数的相应数值, 如表 12 所示:

表 12 节点 $j$ 和节点 $k$ 之间经过节点 $K$ 的路径数 $C_{B}(K)$

\begin{tabular}{cccc}
\hline & $\mathrm{K}$ & $\mathrm{S}$ & $\mathrm{T}$ \\
\hline $\mathrm{A}$ & 3 & 3 & 3 \\
$\mathrm{C}$ & 1 & 1 & 1 \\
$\mathrm{D}$ & 1 & 1 & 1 \\
$\mathrm{E}$ & 1 & 1 & 1 \\
$\mathrm{I}$ & 1 & 1 & 1 \\
$\mathrm{M}$ & 1 & 1 & 1 \\
\hline$(12)$ 节点 $j$ 到节点 $k$ 之间经过节点 $L$ 的路 & 径数的相应数值, 如表 13 所示:
\end{tabular}

(12) 节点 $j$ 到节点 $k$ 之间经过节点 $L$ 的路 径数的相应数值, 如表 13 所示: 
Risk Analysis and Crisis Response in Big Data Era (RAC-16)

表 13 节点 $j$ 和节点 $k$ 之间经过节点 $L$ 的路径数 $C_{B}(L)$

\begin{tabular}{llllllll}
\hline & $\mathrm{L}$ & $\mathrm{N}$ & $\mathrm{P}$ & $\mathrm{Q}$ & $\mathrm{R}$ & $\mathrm{S}$ & $\mathrm{T}$ \\
\hline $\mathrm{A}$ & 2 & 2 & 2 & 2 & 2 & 2 & 6 \\
$\mathrm{D}$ & 1 & 1 & 1 & 1 & 1 & 1 & 3 \\
$\mathrm{E}$ & 1 & 1 & 1 & 1 & 1 & 1 & 3 \\
\hline
\end{tabular}

(13) 节点 $j$ 到节点 $k$ 之间经过节点 $M$ 的路 径数的相应数值, 如表 14 所示:

表 14 节点 $j$ 和节点 $k$ 之间经过节点 $M$ 的路径数 $C_{B}(M)$

\begin{tabular}{cccccccccc}
\hline & $\mathrm{B}$ & $\mathrm{H}$ & $\mathrm{I}$ & $\mathrm{J}$ & $\mathrm{K}$ & $\mathrm{M}$ & $\mathrm{N}$ & $\mathrm{S}$ & $\mathrm{T}$ \\
\hline $\mathrm{A}$ & 2 & 2 & 2 & 2 & 2 & 2 & 6 & 4 & 10 \\
$\mathrm{D}$ & 1 & 1 & 1 & 1 & 1 & 1 & 3 & 2 & 5 \\
$\mathrm{E}$ & 1 & 1 & 1 & 1 & 1 & 1 & 3 & 2 & 5 \\
\hline
\end{tabular}

（14）节点 $j$ 到节点 $k$ 之间经过节点 $N$ 的路 径数的相应数值, 如表 15 所示:

表 15 节点 $j$ 和节点 $k$ 之间经过节点 $N$ 的路径数 $C_{B}(N)$

\begin{tabular}{ccc}
\hline & $\mathrm{N}$ & $\mathrm{T}$ \\
\hline $\mathrm{A}$ & 17 & 17 \\
$\mathrm{~B}$ & 2 & 2 \\
$\mathrm{C}$ & 6 & 6 \\
$\mathrm{D}$ & 5 & 5 \\
$\mathrm{E}$ & 4 & 4 \\
$\mathrm{H}$ & 1 & 1 \\
$\mathrm{I}$ & 3 & 3 \\
$\mathrm{~J}$ & 1 & 1 \\
$\mathrm{~L}$ & 1 & 1 \\
$\mathrm{M}$ & 3 & 3 \\
$\mathrm{~V}$ & 1 & 1 \\
\hline$(15)$ 节点 $j$ 到节点 $k$ 之间经过节点 $O$ 的路 径数的相应数值, 如表 16 所示:
\end{tabular}

表 16 节点 $j$ 和节点 $k$ 之间经过节点 $O$ 的路径数 $C_{B}(O)$

\begin{tabular}{lllllll}
\hline & $\mathrm{O}$ & $\mathrm{P}$ & $\mathrm{Q}$ & $\mathrm{R}$ & $\mathrm{S}$ & $\mathrm{T}$ \\
\hline $\mathrm{A}$ & 2 & 2 & 2 & 2 & 2 & 4 \\
$\mathrm{D}$ & 1 & 1 & 1 & 1 & 1 & 2 \\
$\mathrm{E}$ & 1 & 1 & 1 & 1 & 1 & 2 \\
\hline
\end{tabular}

(16) 节点 $j$ 到节点 $k$ 之间经过节点 $P$ 的路 径数的相应数值, 如表 17 所示:

表 17 节点 $j$ 和节点 $k$ 之间经过节点 $P$ 的路径数 $C_{B}(P)$

\begin{tabular}{llllll}
\hline & $\mathrm{P}$ & $\mathrm{Q}$ & $\mathrm{R}$ & $\mathrm{S}$ & $\mathrm{T}$ \\
\hline $\mathrm{A}$ & 5 & 5 & 5 & 5 & 10 \\
$\mathrm{D}$ & 2 & 2 & 2 & 2 & 4 \\
$\mathrm{E}$ & 2 & 2 & 2 & 2 & 4 \\
$\mathrm{G}$ & 1 & 1 & 1 & 1 & 2 \\
$\mathrm{~L}$ & 1 & 1 & 1 & 1 & 2 \\
$\mathrm{O}$ & 1 & 1 & 1 & 1 & 2 \\
$\mathrm{U}$ & 1 & 1 & 1 & 1 & 2 \\
\hline
\end{tabular}


Risk Analysis and Crisis Response in Big Data Era (RAC-16)

（17）节点 $j$ 到节点 $k$ 之间经过节点 $Q$ 的路 径数的相应数值, 如表 18 所示:

\begin{tabular}{cccc} 
& \multicolumn{2}{c}{ 表 18 节点 $j$ 和节点 $k$ 之间经过节点 $Q$ 的路径数 $C_{B}(Q)$} \\
\hline $\mathrm{A}$ & $\mathrm{Q}$ & $\mathrm{R}$ & $\mathrm{T}$ \\
$\mathrm{D}$ & 6 & 6 & 6 \\
$\mathrm{E}$ & 2 & 2 & 2 \\
$\mathrm{G}$ & 2 & 2 & 2 \\
$\mathrm{~L}$ & 2 & 2 & 2 \\
$\mathrm{O}$ & 1 & 1 & 1 \\
$\mathrm{P}$ & 1 & 1 & 1 \\
$\mathrm{U}$ & 1 & 1 & 1 \\
\hline
\end{tabular}

（18）节点 $j$ 到节点 $k$ 之间经过节点 $R$ 的路 径数的相应数值, 如表 19 所示:

表 19 节点 $j$ 和节点 $k$ 之间经过节点 $R$ 的路径数 $C_{B}(R)$

\begin{tabular}{ccc}
\hline & $\mathrm{R}$ & $\mathrm{T}$ \\
\hline $\mathrm{A}$ & 6 & 6 \\
$\mathrm{D}$ & 2 & 2 \\
$\mathrm{E}$ & 2 & 2 \\
$\mathrm{G}$ & 2 & 2 \\
$\mathrm{~L}$ & 1 & 1 \\
$\mathrm{O}$ & 1 & 1 \\
$\mathrm{P}$ & 1 & 1 \\
$\mathrm{Q}$ & 1 & 1 \\
$\mathrm{U}$ & 1 & 1 \\
\hline$(19)$ 节点 $j$ 到节点 $k$ 之间经过节点 $S$ 的路 $\quad$ 径数的相应数值, 如表 20 所示:
\end{tabular}

表 20 节点 $j$ 和节点 $k$ 之间经过节点 $S$ 的路径数 $C_{B}(S)$

\begin{tabular}{lcc}
\hline & S & T \\
\hline A & 15 & 15 \\
B & 1 & 1 \\
C & 3 & 3 \\
D & 5 & 5 \\
E & 4 & 4 \\
F & 1 & 1 \\
G & 1 & 1 \\
I & 2 & 2 \\
J & 1 & 1 \\
K & 1 & 1 \\
L & 1 & 1 \\
M & 2 & 2 \\
O & 1 & 1 \\
P & 1 & 1 \\
U & 1 & 1 \\
\hline
\end{tabular}


Risk Analysis and Crisis Response in Big Data Era (RAC-16)

（20）节点 $j$ 到节点 $k$ 之间经过节点 $T$ 的 路径数的相应数值，如表 21 所示:

表 21 节点 $j$ 和节点 $k$ 之间经过节点 $T$ 的路径数 $C_{B}(T)$

\begin{tabular}{cc}
\hline & T \\
\hline A & 38 \\
B & 3 \\
C & 9 \\
D & 12 \\
E & 10 \\
F & 1 \\
G & 3 \\
H & 1 \\
I & 5 \\
J & 2 \\
K & 1 \\
L & 3 \\
M & 5 \\
N & 1 \\
O & 2 \\
P & 2 \\
Q & 1 \\
R & 1 \\
S & 1 \\
U & 3 \\
V & 1 \\
\hline$(21)$ 节点 $j$ 到节点 $k$ 之间经过节点 $U$ 的路 & 径数的相应数值, 如表 22 所示: \\
&
\end{tabular}

表 22 节点 $j$ 和节点 $k$ 之间经过节点 $U$ 的路径数 $C_{B}(U)$

\begin{tabular}{cccccc}
\hline & $\mathrm{P}$ & $\mathrm{Q}$ & $\mathrm{R}$ & $\mathrm{T}$ & $\mathrm{U}$ \\
\hline $\mathrm{A}$ & 1 & 2 & 2 & 2 & 1 \\
$\mathrm{G}$ & 1 & 2 & 2 & 2 & 1 \\
\hline
\end{tabular}

(22) 节点 $j$ 到节点 $k$ 之间经过节点 $V$ 的路 径数的相应数值, 如表 23 所示:

表 23 节点 $j$ 和节点 $k$ 之间经过节点 $V$ 的路径数 $C_{B}(V)$

\begin{tabular}{llll}
\hline & $\mathrm{N}$ & $\mathrm{T}$ & $\mathrm{V}$ \\
\hline $\mathrm{A}$ & 1 & 1 & 1 \\
$\mathrm{D}$ & 1 & 1 & 1 \\
\hline
\end{tabular}

利用模型公式 (4)，可得出相关节点 $A$ 不纳入计算范围内），如表 5-24 所示。 流介数指标数值 (作为始端节点的洪涝灾害 
Risk Analysis and Crisis Response in Big Data Era (RAC-16)

\begin{tabular}{ccc} 
& \multicolumn{2}{c}{ 表 24 各个节点的流介数指标数值 } \\
\hline B & 对应社会稳定风险 & $\boldsymbol{C}_{\boldsymbol{B}}(\boldsymbol{x})$ \\
$\mathrm{C}$ & 经济损失 & 15.3341 \\
$\mathrm{D}$ & 房屋倒塌 & 1.8782 \\
$\mathrm{E}$ & 电力设施破坏 & 3.0343 \\
$\mathrm{~F}$ & 交通受阻中断 & 12.2818 \\
$\mathrm{G}$ & 通讯中断 & 1.2346 \\
$\mathrm{H}$ & 农作物受损 & 1.5061 \\
$\mathrm{I}$ & 灾民居无住所 & 5.4388 \\
$\mathrm{~J}$ & 人员伤亡 & 15.6232 \\
$\mathrm{~K}$ & 犯罪率上升 & 7.6175 \\
$\mathrm{~L}$ & 传染病流行 & 4.3784 \\
$\mathrm{M}$ & 人员滞留 & 4.4628 \\
$\mathrm{~N}$ & 交通事故 & 9.7580 \\
$\mathrm{O}$ & 人员心理影响 & 8.8154 \\
$\mathrm{P}$ & 物资运输困难 & 4.0610 \\
$\mathrm{Q}$ & 物资缺乏 & 13.9482 \\
$\mathrm{R}$ & 物价上涨 & 9.5956 \\
$\mathrm{~S}$ & 哄抢购物 & 6.4289 \\
$\mathrm{~T}$ & 社会大众恐慌 & 11.0890 \\
$\mathrm{U}$ & 流言及群体行为 & 10.5000 \\
$\mathrm{~V}$ & 市场供应紧张 & 3.2930 \\
$\mathrm{~N}$ & 生产生活困难 & 1.1842
\end{tabular}

从表 24 可以看出, 在整个洪涝灾害社 会稳定风险结构中，人员伤亡、经济损失、 物资缺乏、交通受阻中断、社会大众恐慌、 流言及群体行为在整个洪涝灾害社会稳定 风险结构中所处位置是最终为重要的; 交通 事故、物价上涨、人员心理影响、犯罪率上 升、哄抢购物、灾民居无住在整个洪涝灾害 社会稳定风险结构中所处位置次之, 人员滞 留、传染病流行、物资运输困难、市场供应 紧张、电力设施破坏、房屋倒塌、农作物受 损、通讯中断、生产生活困难在整个洪涝灾 害社会稳定风险结构中所处位置最后。

在洪涝灾害社会稳定风险评价的过程 中, 针对某一风险评价时, 不该局限于其所 引起的直接损害, 应着眼于该风险在整个洪 涝灾害社会稳定风险结构中所处位置, 即应 该考虑其所能引起的后续损害。

\section{5. 结论}

重大洪涝灾害的发生, 不仅带来了生命 经济损失, 同时还造成了社会稳定风险, 严 重制约着社会的发展进步。在对湖南省怀化
市 2013 年 8 月的重大洪涝灾害调研的基础 上, 综合相关文献, 得出了重大洪涝灾害社 会稳定风险拓扑图, 在此基础上, 着眼于该 风险在整个洪涝灾害社会稳定风险结构中 所处位置, 将复杂网络中的流介数指标模型 加以改进, 对特大洪涝灾害社会稳定风险评 价进行了分析。分析结果表示, 在整个洪涝 灾害社会稳定风险结构中，人员伤亡、经济 损失、物资缺乏、交通受阻中断、社会大众 恐慌、流言及群体行为、洪涝灾害本身在整 个洪涝灾害社会稳定风险结构中所处位置 是最为重要的; 交通事故、物价上涨、人员 心理影响、犯罪率上升、哄抢购物、灾民居 无住所在整个洪涝灾害社会稳定风险结构 中所处位置次之, 人员滞留、传染病流行、 物资运输困难、市场供应紧张、电力设施破 坏、房屋倒塌、农作物受损、通讯中断、生 产生活困难在整个洪涝灾害社会稳定风险 结构中所处位置最后。 


\section{参考文献}

[1] Hu Haibo and Zhang Yan-li. Quick Assessing Model on Casualty Loss in Rainstorms. Journal of Catastrophology, 2014, 29( 1) : 30-36.

[2] Zhang Dongdong,Yan Denghua,Wang Yicheng,et al. Research Progress on Risk Assessment and Integrated Strategies for Urban Pluvial Flooding . Journal of Catastrophology, 2014, 29(1):144-149.

[3]JING Fengwei, YANG Yongguo, DENG Shizan,et al. Simulation and evaluation of flood disaster. Geospatial Information, 2011, 9( 4) : 122-124.

[4]FU Yicheng, WEI Chuanjiang, WANG Qimeng,et al. Study on regional flood risk assessment system. Journal of Catastrophology, 2009, 24(3) : 27-32.

[5]LI Guofang,ZHENG Lingyu,TONG Yiyi,et al. Effects evaluation of urbanization on flood risk in the Yangtze River Delta. Resources and Environment in the Yangtze Basin, 2013,22( 3 ) : 386-391.

[6] Hall J W,Sayers P B,Dawson R J. National-scale assessment of current and future flood risk in England and Wales. Natural Hazards, 2005,36: 147-165.

[7] Dilley M,Chen R S,Deichmann U,et al. Natural Disaster Hotspots: A Global Risk Analysis. Washington: The World Bank, 2005.

[8]ZHANG You,WANG Shaoqiang,GE Quansheng,et al. Risk assessment of flood disaster in Jiangxi Province based on GIS . Resources and Environment in the Yangtze Basin 2011,20(21) : 166-172.

[9]LIU Jiafu,LI Jing,LIANG Yuhua,et al. Storm flood risk assessment in the typical regions of Asia . Scientia Geographica Sinica, 2011,31( 10) : 1266-1271 .

[10]MA Dingguo, LIU Ying, CHEN Jie, et al. Farmers' Vulnerability to Flooding in the Poyang Lake Region. Acta Geographica Sinica, 2007,62( 3) : 321-332.

[11] Tawatchai T,Mohammed F K. Flood hazard and risk analysis in the southwest region of Bangladesh . Hydrological Process, 2005( 19) : 2055-2069.

[12] Xilei Pang . Dynamic Vulnerability Analysis of Population for Flood Disaster in Urban Area. Journal of Risk Analysis and Crisis Response,2013, 3(4):166-174.

[13]HUANG Minsheng, HUANG Chengcheng. Research on grade model of flood risk assessment . Journal of Catastrophology, 2007,22( 1) : 1-5.

[14]YANG Lechan,DENG Song,XU Jianghui. Flood risk evaluation algorithm on BP Net . Computer Technology and Development, 2010,20( 4) : 232-234.

[15]LAI Chengguang,WANG Zhaoli,SONG Haijuan. risk assessment of flood hazard in Beijiang River Basin Based on BP Neural Network. Water Resources and Power, 2011,29(3) : 57-59.

[16] 李帅杰,李昌志,程晓陶. 区域洪灾风险 评价方法初探一以浙江省为例. 水利水 电技术, 2012,43( 3) : 82-87.

[17] DAI Juan,PAN Yinong,LIU Qing,et al. Application of improved AHP in county-scale rainstorm and flood risk assessment. Journal of the Meteorological Sciences, 2014,34( 4) : 428-434.

[18]WANG Zhaoli,LAI Chengguang,CHEN Xiaohong. Spatially fuzzy comprehensive assessment model for flood hazardrisk based on entropy weight. Journal of Hydroelectric Engineering,2012,31( 5) : 35-40.

[19]宁娜,舒和平,刘东飞,马金珠. 基于熵权 和模糊评判的单沟泥石流危险性评价 $[\mathrm{J}]$ 兰州大学学报 (自然科学 版),2014,50(3):369-375.

[20]ZOU Qiang,ZHOU Jiangzhong, ZHOU Chao, et al. Flood disaster risk analysis based on principle of maximum entropy, 
Risk Analysis and Crisis Response in Big Data Era (RAC-16)

and attribute interval recognition theory. Advances in Water Science, 2012, 23( 3) : 323-333.

[21] Xianfu Cheng,Honghu Sun,et al. Flood Disaster Risk Assessment and Spatial Distribution Characteristics along the Yangtze River in Anhui Province t. Journal of Risk Analysis and Crisis Response,
2014, 4(4) :238-242.

[22]Newman M E J. A measure of betweenness centrality based on random walks. Social Networks,2005,27(1):39-54. [23]Freeman L C, Borgatti S P, White D R. Centrality in valued graphs: A measure of betweenness based on network flow. Social Networks, 1991,13(2):141-154. 